\title{
Utilisation de la simulation dans des projets de robotique ${ }^{1}$
}

\author{
Antoine TAuvel (ENSEA Cergy-Pontoise²), Nicolas Simond (Lycée Langevin Wallon, Champigny sur Marne), \\ Sylvain Todeschini (Lycée Simone de Beauvoir, Garges-lès-Gonesses). \\ tauvel@ensea.fr, nicolas.simond@gmail.com, sylvaintodeschini@hotmail.fr
}

RESUME : Le département GEII de l'iut de Sarcelles propose à ses étudiants un projet universitaire durant leur première année de formation, qui débouche sur une compétition amicale de robotique. Dans ce cadre, les auteurs ont développé un simulateur simple de stratégie de robot afin d'aider à la compréhension des mécanismes de prise de décision. Ce simulateur, basé sur le logiciel de calcul scientifique SciLab, est disponible en téléchargement sur le site : http://www.tauvel.net rubrique téléchargement. L'expérience pédagogique menée est proche de celle de l'iut de Cachan, mais s'en différencie en ce que le projet n'est pas le support unique de l'enseignement scientifique et technologique.

Mots clés : Robotique, Simulateur, DUT GEII, expérience d'apprentissage par projet.

\section{INTRODUCTION}

"Motiver les étudiants »! Tel serait en fin de compte le sous-titre de la quasi-totalité des articles contenus dans ces actes, tant il est vrai que l'intérêt pour la matière détermine la réussite des étudiants. Les approches par projet ont depuis longtemps fait la preuve de leur intérêt pour obtenir cette motivation. Dans ces approches, la question du rapport entre les enseignements théoriques et les enseignements de projet est fondamentale. Le but de cet article est de présenter l'expérience du site de Sarcelles en la matière. Cette expérience est à rapprocher de celle de l'iut de Cachan, présentée lors du colloque du GESI de Lyon en juin 2005 (les actes, paru en décembre 2005, présentent partiellement la démarche de ce département [1]).

\section{CONTEXTE PÉDAgOGIQUE}

\subsection{Contexte général}

Depuis 40 ans, les formations de DUT GEII (génie électrique et informatique industrielle) forment en deux ans des techniciens pour l'industrie électronique. Comme de nombreuses formations scientifiques et technologiques en France, elles perdent chaque année en attractivité et en sélectivité. Dans ces conditions, nombreux sont les départements qui cherchent la «formule magique » pour éveiller l'intérêt et la créativité des étudiants.

Le département GEII de Sarcelles, dépendant de l'IUT de Cergy-Pontoise, n'échappe bien sûr pas à ce phénomène. Aussi y a-t-on mené de nombreuses expériences (semestrialisation, groupes de niveaux, module d'apprentissage adapté...). L'échec se situant

majoritairement en première année ${ }^{3}$, nous avons décidé de faire un effort pédagogique et financier portant spécifiquement sur cette année de formation.

Le principe essentiel a été de regrouper les trois « Études et réalisations » ponctuelles dans l'année en un seul projet de volume horaire conséquent. Cent heures / étudiant ont ainsi été dispensées entre Novembre et Juin, à raison d'une demi-journée par semaine exclusivement consacrée à un projet unique. Ce projet doit servir de support d'enseignement à un maximum de compétences technologiques.

\subsection{La robotique comme catalyseur d'intérêt}

Une fois posé le principe de la réforme, reste à trouver LE sujet permettant tout à la

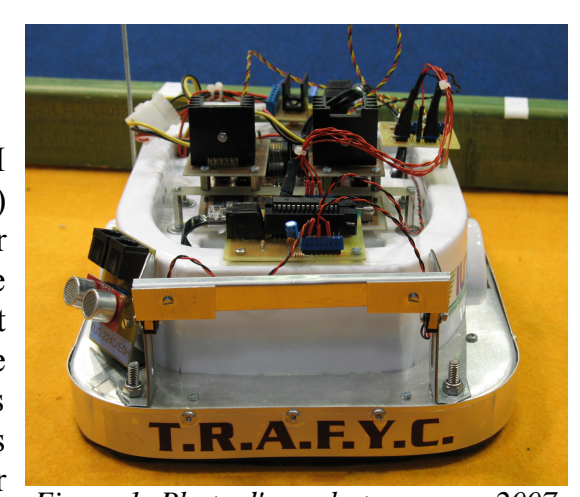

Figure 1: Photo d'un robot conçu en 2007 fois de mettre en application un maximum de concepts théoriques et d'éveiller la curiosité des étudiants. Nous avons choisi la robotique mobile. Outre l'aspect ludique et la grande variété de thèmes abordables, l'indéniable essor économique de la robotique est un facteur de motivation. On a par exemple transmis aux étudiants une copie de [2], afin de souligner, ne serait-ce qu'à travers la personnalité de l'auteur, les enjeux économiques liés à la discipline.

3 A titre d'exemple, le taux de passage entre la première et la deuxième année en 2006, année « record», a été de $37 \%$. Il se situe plus classiquement autour de $50 \%$. L'essentiel est dû à des abandons en cours d'année scolaire.

1 Les auteurs tiennent à exprimer leur gratitude au consortium Scilab. Grâce à l'écoute et la disponibilité de ses directeurs MM. Gomez et Halgand nous avons pu rencontrer MM. Jreige et Silvy. Leurs conseils ont rendus le code de départ fiable et performant (notamment en terme de vitesse de simulation).

2 L'auteur, passé au 1er septembre 2008 de l'iut de Cergy-Pontoise sur le site de Sarcelles à l'ENSEA, tient ici à remercier cette dernière pour son soutien financier à cet article, basé sur une expérience menée à l'iut. 
Les 53 départements GEII de France organisent chaque année à Vierzon un concours de robotique ${ }^{4}$, a priori à destination des étudiants de deuxième année. Le concours est réservé aux étudiants d'IUT, car son niveau est trop simple pour une équipe d'étudiantsingénieurs. A titre d'exemple, 33 robots venant de 24 départements se sont affrontés en 2007.

Ce concours nous a fourni la base du cahier des charges, que nous avons simplifié en fonction des connaissances des étudiants. Une compétition interne au département de Sarcelles a été organisée. D'autre part, le robot le plus «méritant» (au sens des enseignants, c'est à dire pas forcément le plus performant) a été envoyé au concours national de Vierzon. Le concept même de compétition crée ainsi une saine émulation entre les groupes étudiants.

Les étudiants ont été regroupé par 3 ou 4, essentiellement en fonction de leurs affinités. Quinze robots ont été mis en chantier, encadrés par les trois auteurs.

\subsection{Evaluation des étudiants.}

Ce projet compte pour un poids important dans le diplôme. Il a été évalué six fois (trois à chaque semestre), sous la forme d'une note de réalisation, d'une note de rapport et d'une note de soutenance orale.

\section{CAHIER DES CHARGES}

Le robot à construire est fortement inspiré du concours national de robotique GEII [3], avec un cahier des charges simplifié. Il s'agit essentiellement d'un robot suiveur de ligne (ligne blanche sur fond bleu). La base mécanique est imposée, ainsi que la motorisation et les batteries $^{5}$. Le gabarit maximum est de 30 sur $50 \mathrm{~cm}$. Le rayon de courbure minimal de la piste est de $30 \mathrm{~cm}$. Deux robots ne peuvent pas se croiser, mais une piste peut se croiser elle-même (il s'agit de la principale modification du cahier des charges original). Des marqueurs spécifiques indiquent au robot la possibilité de faire un "stop figure », un tour complet sur lui-même qui permet d'engranger un bonus de temps. Le départ peut avoir lieu en aveugle, ce qui donne là encore un bonus.

Nous avons ajouté une contrainte forte, compte tenu du mode de conception de nos robots : chaque robot devait être absolument unique, de manière à stimuler la créativité des étudiants (capacité à notre sens aussi indispensable à un électronicien que la rigueur). Aussi

4 Site officiel du concours de robotique GEII : $\underline{\mathrm{http}: / /}$ web.geii.univ-savoie.fr/geii/robots/robots.htm

5 On peut se procurer un kit à coût modique auprès de l'iut de cachan, à l'adresse citée dans [3]. avons-nous croisé les critères indiqués dans le tableau 1, ce qui permet de définir au moins huit types de robots différents (16 si l'on tient compte des hacheurs boost en option).

Les différences de conception secondaires viennent ensuite du choix des microprocesseurs (3 microprocesseurs, de générations diverses, étaient disponibles), ainsi que de la vision. Si la plupart des étudiants ont choisi les capteurs reflectométriques, le nombre et l'implantation des capteurs a donné lieu à de nombreuses variations (de 4 à 8 capteurs, positionnés de manière très variable). Au total, 15 robots ont été conçus en 2008, chacun effectivement nettement différencié des autres.

Les langages de programmation utilisés par les étudiants sont le langage $\mathrm{C}$ (pour les microcontrôleurs) et le VHDL pour ceux qui ont choisi la version CPLD.

\begin{tabular}{|l|l|l|}
\hline Type de vision & $\begin{array}{l}\text { Capteurs } \\
\text { reflectomètriques }\end{array}$ & Caméra \\
\hline $\begin{array}{l}\text { Type de } \\
\text { hacheur }\end{array}$ & $\begin{array}{l}\text { Intégré 4 } \\
\text { quadrants }\end{array}$ & $\begin{array}{l}\text { Discret un } \\
\text { quadrant }\end{array}$ \\
\hline $\begin{array}{l}\text { Implantation de } \\
\text { stratégie }\end{array}$ & $\begin{array}{l}\text { Microprocesseurs } \\
8 \quad \text { bits (trois } \\
\text { modèles } \\
\text { disponibles) }\end{array}$ & $\begin{array}{l}\text { CPLD } \\
\text { (programmé en } \\
\text { VHDL) }\end{array}$ \\
\hline Optionnel : & $\begin{array}{l}\text { Hacheur boost } \\
\text { pour augmenter la } \\
\text { vitesse }\end{array}$ \\
\hline
\end{tabular}

Tableau 1: Principaux choix de conception.

\section{SYNCHRONISATION DES ENSEIGNEMENTS}

La difficulté majeure dans la mise au point du projet pédagogique a été de synchroniser les enseignements « classiques » et les enseignements de projet. Le projet a démarré fin novembre, peu de temps après l'étude en cours de la diode. Les premières séances ont été consacrées à des réflexions générales sur la gestion de projet et le cahier des charges, ainsi qu'au relevé de caractéristiques des capteurs photométriques. Seuls trois groupes d'étudiants volontaires ont choisi les caméras. Ils ont donc dû se renseigner sur les signaux composites.

Une seule notion a dû faire l'objet d'un traitement spécifique : la conversion d'énergie. En effet, les hacheurs sont abordés en temps normal en deuxième année. Nous avons mis au point, avec l'accord de l'enseignant responsable, une séance comportant une part non négligeable de théorie. 
Nous avons choisi de découper le projet en trois thèmes, abordés dans l'ordre :

- Vision : principes de capteurs simples, relevé de caractéristiques, apprentissage des principes de routage sur une carte simple (sans horloge, donc sans risques de diaphonies). Ce thème a duré, en moyenne, de Novembre à fin Janvier. Trois groupes volontaires ont choisi une caméra, orientant leurs premières séances vers un travail plus « cérébral» de compréhension des trames d'un signal composite.

- Puissance : découverte des hacheurs, comparaisons aux solutions linéaires, type ballast, lecture de datasheet (choix du bras de pont). Ce thème a duré en moyenne de Janvier à Mars.

- Stratégie : mise en application du cours d'informatique industrielle, utilisation d'un simulateur pour le dimensionnement des réponses. Les binômes les plus avancés ont pu réfléchir à la notion de régulation de vitesse. Ce thème a occupé les étudiants de Mars à Juin.

Le concours national GEII a lieu le premier week-end de juin, la compétition interne de Sarcelles a été fixée à la mi-juin, le dernier jour des cours officiels. Le concours interne reconnaît trois vainqueurs : le robot le plus rapide, le robot le plus soigné, et le robot le plus drôle. Une récompense modique, financée par le $\mathrm{BdE}$ de l'iut, a été attribuée à chacun des vainqueurs.

\section{SIMULATION}

A quoi sert une simulation ? Nous autres enseignants, chercheurs et ingénieurs répondons tous en cœur : «à réduire les coûts de conception et diminuer le $\mathrm{TTM}^{6} »$. Nous proposons ici une fonction supplémentaire, pédagogique. En effet, dès les premières séances, la question de la stratégie de commande a été posée par les étudiants, qui ne voyaient pas comment faire le lien entre

l'information

visuelle et la
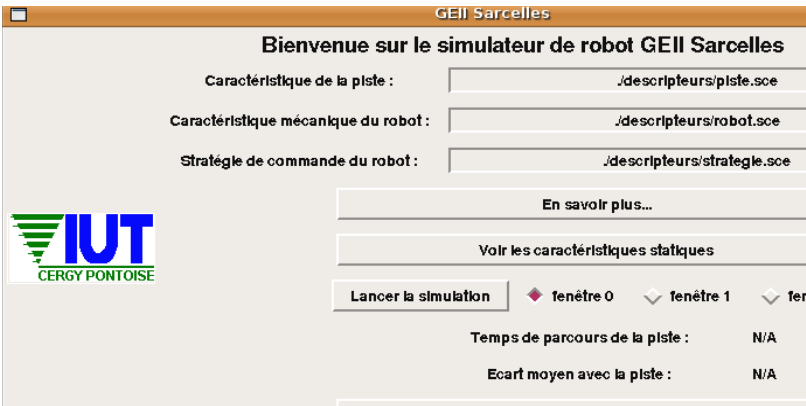

En simulation, le capteur caméra est perçu comme un méga opto-coupleur amélioré : chaque pixel d'une ligne de l'image est assimilé à un opto-coupleur. L'information recueillie après seuillage des niveaux de gris que forme une ligne de l'image est beaucoup plus précise que celle que fournissent quelques optocoupleurs, du fait du pas d'échantillonnage beaucoup plus petit. Il est alors possible de déterminer la position relative du robot par rapport à la médiane de la piste.

cette stratégie afin d'aider à la compréhension des enjeux.

\subsection{Outils de simulation}

Poussés par des instincts culturels et économiques bien libres multiplato avons donc retenu $\mathrm{SciLab}^{7}$, logiciel libre de calcul scientifique matriciel doté d'un interpréteur Tcl/Tk pour la gestion de l'interface graphique.

\subsection{Les capteurs dans le simulateur}

La piste suivie par le robot est détectée à partir soit d'opto-coupleurs répartis sur le châssis, soit d'un traitement rudimentaire de l'image fournie par une camera embarquée. De manière générale, les capteurs présence de la piste : un capteur sera dit «éclairé 》 si un point de la piste se situe à moins de largeur_piste de ce dernier.

Le nombre et la disposition des opto-coupleurs sous le châssis du robot doivent répondre à un compromis coût / efficacité que la simulation permet en partie d'éclaircir.

Une solution alternative, qui présente de nombreux avantages, est d'embarquer une caméra centrée dans l'axe longitudinal du robot qui observe principalement le plan de la piste devant le robot.

Autre avantage non Autre avantage non
négligeable de l'emploi d'une caméra centrée et orientée dans l'axe longitudinal du robot : les capteurs sont déportés devant le robot, ce qui permet d'anticiper la trajectoire à suivre. Le paramètre

d'anticipation peut être facilement réglé en
Volr les caractérlstiques statkques

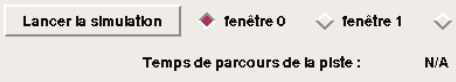

Ecart moyen avec b plste: N/A VIsuallser les vitesses commande des moteurs. Face à des questions récurrentes s'est peu à peu imposée l'idée de simuler

6 Time To Market. 
déterminant quelle est la ligne de l'image sur laquelle sera effectué le traitement. Il ne faut pas oublier de prendre en compte l'effet perspectif de la caméra. La piste perçue avec une caméra assimilée à son modèle « pinhole » s'avère être l'intersection d'un cône issu du foyer de la caméra avec un plan. Dès lors, il faut prévoir que plus la ligne de l'image est haute dans l'image, plus le pas entre 2 pixels augmente.

\subsection{Modèle mécanique}

Le modèle mécanique retenu est une linéarisation des déplacements du mobile, calculée à partir de son centre de gravité. Les deux moteurs imposent un mouvement combiné de translation et de rotation, calculé par pas de une milliseconde.

Le gestion de la réponse mécanique est élémentaire : nous appliquons un modèle du premier ordre à la fonction de transfert entre le rapport cyclique du hacheur et la vitesse des moteurs. La constante de temps appliquée, paramétrable par l'utilisateur, est (pour le moment) la même en accélération ou en décélération, ce qui est la principale approximation de ce modèle.

\subsection{Mode d'emploi succinct}

Un mode d'emploi complet, comprenant notamment un dictionnaire des données à remplir, est inclus dans le téléchargement du logiciel.

Le logiciel fourni est entièrement piloté par une interface graphique sous $\mathrm{Tcl} / \mathrm{Tk}^{8}$. La fenêtre qui apparaît après avoir tapé la commande exec('run.sce') sous Scilab permet de :

- caractériser les différents paramètres que sont la piste, le robot et la commande implémentée,

- afficher sous une même fenêtre graphique les paramètres précédents,

- lancer la simulation puis suivre la trajectoire suivie par le robot au cours du temps ainsi que l'évolution des variables internes tels que les capteurs éclairés, la position relative de la piste retenue et les commandes imposées à chacun des deux moteurs.

La mise en oeuvre d'une simulation requiert une phase d'initialisation des paramètres fournis par défaut. Lors de cette phase, les structures Piste, Robot et Pose doivent être complétées pour les 2 premières et initialisée pour la troisième.

8 http://wfr.tcl.tk/

\subsubsection{Caractéristiques statiques}

La structure Piste contient les informations topographiques du plan de la piste exprimées en centimètres, les coordonnées des points qui forment la piste, sa largeur ainsi que la durée maximum de la simulation exprimée en milliseconde. Trois exemples de pistes de difficulté croissante sont proposés.

La structure Robot contient les côtes, un booléen qui précise le type de capteur employé (caméra ou capteur reflectomètrique) ainsi que son nombre, la constante de temps électromécanique des moteurs exprimée en [ms], le taux de conversion de chaque moteur exprimé en $[\mathrm{cm} / \mathrm{s}]$, la loi de commande de chaque moteur en fonction de la position relative de la piste détectée par les capteurs, les coordonnées homogènes des quatre coins du châssis ainsi que celles des capteurs. Deux exemples de fichiers sont fournis : robot avec optocoupleurs et robot avec caméra.

Il est à noter que le champ relatif aux lois de commande des moteurs est complété par l'appel automatique à la fonction strategie.sce qui, entre autres, concatène les vecteurs lignes alpha_gauche et alpha_droite en une matrice 2 lignes, $\mathrm{n}$ colonnes où chaque valeur correspond à un rapport cyclique de commande de moteur.

Cette même fonction initialise la structure Pose qui contient les champs relatifs aux variables internes et externes au robot. Ces variables évoluent au cours de son déplacement. Parmi les différents champs complétés à chaque itération se trouvent la position courante du robot sur la piste ainsi que son orientation exprimée en radians, les capteurs éclairés et le capteur retenu pour déterminer dans la loi de commande les

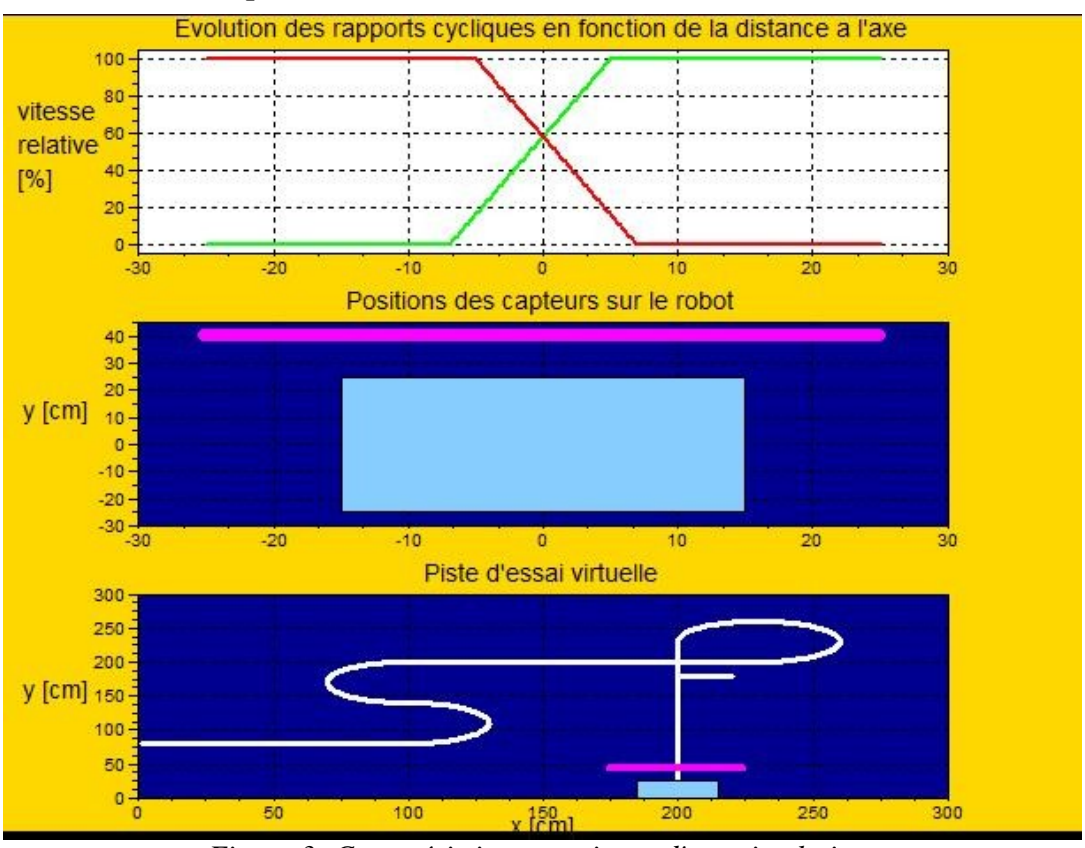

Figure 3: Caractéristiques statiques d'une simulation. 
rapports cycliques de chaque moteur, la vitesse de consigne et instantanée de chaque moteur, les valeurs des déplacements élémentaires (translation selon l'axe longitudinal et amplitude de la rotation).

Le bouton «Voir les caractéristiques statiques» de l'interface affiche sur une même fenêtre graphique les lois de commande des moteurs gauche et droit, la disposition des capteurs en fonction du châssis du robot ainsi que la piste sélectionnée. Elle permet à l'utilisateur de vérifier la cohérence des variables qu'il a modifiées. Vous pouvez en admirer un exemplaire en figure 3.

\subsection{Utilisation pédagogique}

Les étudiants du cursus GEII n'ont pas à priori à leur programme l'utilisation de logiciels de calcul scientifique. Aussi avons-nous conçu le code de façon à ce qu'un minimum de connaissances dans ce domaine soit requis. Les étudiants ont eu besoin d'une à deux heures de préparation pour comprendre les syntaxes de base : notamment les différentes manières de définir une matrice. Cette préparation s'est appuyée sur le mode d'emploi disponible dans le dossier de téléchargement du simulateur.

\subsubsection{Trajectographie}

Le bouton «Lancer la simulation » de l'interface calcule la trajectoire suivie par le robot, puis affiche une animation du déplacement du robot sur la piste ainsi que l'évolution au cours du temps des capteurs éclairés pary $[\mathrm{cm}$ la piste, le capteur retenu pour déterminer la commande des moteurs et les vitesses instantanées et de consigne de chacun des moteurs.

Le pseudo-code de la fonction principale qui remplit la structure Pose est fondé sur une boucle while qui vérifie que le temps
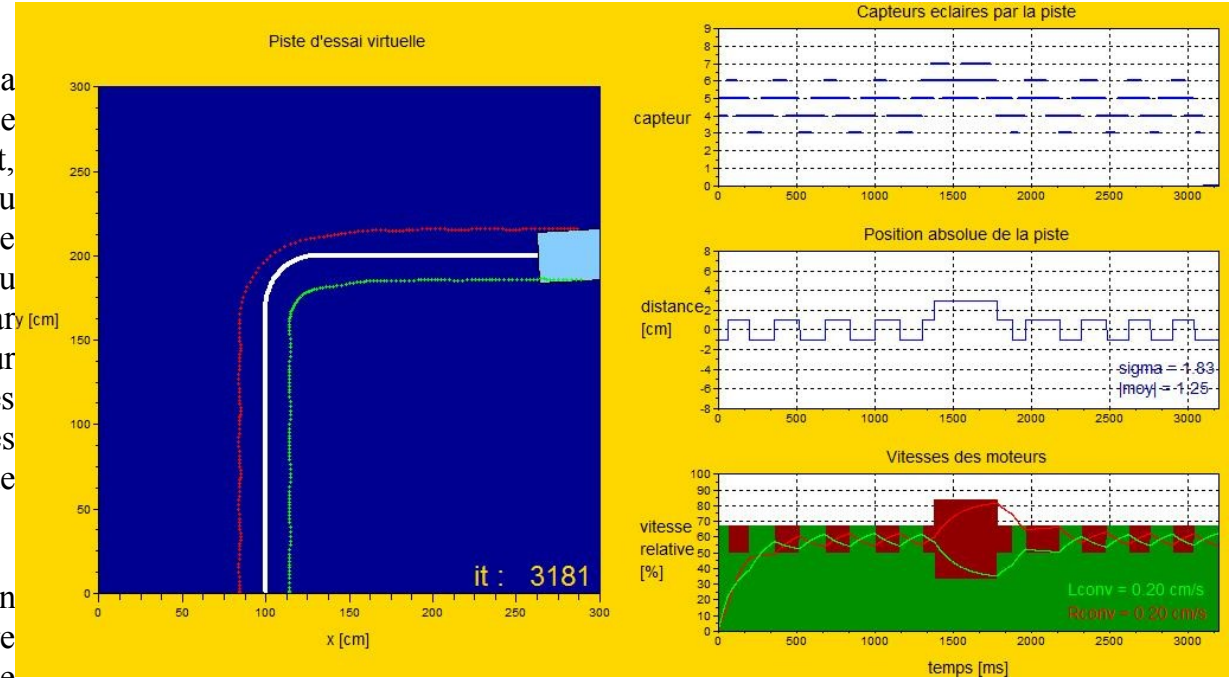

Figure 4: Résultats de simulation pour un robot simple et une piste courbée à $90^{\circ}$.

Notre simulateur ne prétend pas à l'exactitude (voir le paragraphe 5.7). Son but premier est pédagogique. Les objectifs que nous nous étions fixés, et que nous estimons atteints, sont les suivants :

- Comprendre la conception de la loi de commande du robot.

- Illustrer le dilemme entre rapidité et précision dans le suivi de la trajectoire.

- Illustrer l'importance de l'inertie, avec la possibilité de faire varier la constante de temps.

- Guider les étudiants dans leurs choix d'implantation des capteurs.

- Illustrer l'importance d'une régulation de vitesse, avec la possibilité de fixer des vitesses maximales différentes sur les moteurs gauche et droit.

La figure 4 présente un résultat de simulation normal pour un robot simple ( 8 capteurs) et une piste représentant une simple courbure à $90^{\circ}$. On notera la visualisation des vitesses, en bas à droite, faisant clairement apparaître le virage et l'effet de l'inertie.

\section{6 « Licence » d'utilisation}

Vous trouverez le simulateur disponible sur le site : http://www.tauvel.net/ rubrique téléchargement.

Code 1 : pseudo-code de la fonction de simulation. 
Ce travail est totalement libre de droit. Les collègues souhaitant travailler avec ce logiciel n'ont d'autre obligation que de ne pas modifier le bouton « en savoir plus » de l'interface signalant le lieu et les noms des premiers concepteurs. Nous sommes évidemment ouverts à toute suggestion d'amélioration.

\subsection{Validation du modèle}

Notre simulateur fonctionne correctement, mais quelle est sa précision ? Cette question, fondamentale dans le cas d'un «vrai » simulateur, a été laissée de côté par les auteurs faute de temps, au profit de l'interactivité de l'outil. Nous ne manquerons toutefois pas l'occasion de tester les résultats de simulations lors des prochains essais.

\section{BILAN DE L'EXPÉRIENCE}

Le concours interne de robotique de l'institut, qui aurait dû voir s'affronter quinze robots n'a permis de n'en voir évoluer que sept. Pour la première année d'expérimentation, sans doute avons nous laissé trop d'autonomie aux étudiants par rapport aux difficultés prévisibles.

Malgré cela, les étudiants ont fortement appréciés cette expérience. Le concours, doté de quelques prix financés par le bureau des élèves, a permis en fin d'année de voir la cohésion que l'ensemble de la promotion à acquise via le projet. La possibilité de simuler le comportement du robot à permis d'aller plus vite sur lors de la phase de programmation de la stratégie.

L'investissement financier et temporel dans le projet est rentabilisé par une forte réutilisabilité dans les années à venir, dans le cadre d'une refonte de la pédagogie de la première année du département GEII de Sarcelles.

\section{CONCLUSION}

La mise au point d'un simulateur fiable a un intérêt pédagogique certain. En effet, les interrogations des étudiants sont nombreuses quant à la stratégie de commande. Par exemple, l'une des confusions les plus fréquentes était que puisque le robot doit rester au milieu de la piste, il suffit d'un seul capteur au milieu de la piste !!! Cet outil a fourni une réponse adaptée et personnalisée à ces interrogations. Malheureusement, les premières versions n'ont pas été prêtes assez tôt pour une utilisation en optimisation de la géométrie des capteurs.

Par contre, l'objectif de motivation des étudiants est un demi-échec. Le taux d'abandon en cours d'année n'a pas été plus faible lors de l'expérimentation que les années passées. On a constaté malgré tout sur la promotion 2006-2007 ayant bénéficié des premières esquisses de cette expérience un fort regain de motivation pour l'étude des systèmes embarqués. Traditionnellement faiblement représenté dans les profils de poursuite d'étude de nos étudiants, cette discipline a attiré un peu moins de la moitié de la promotion dans des cursus variés allant $\mathrm{du} \mathrm{Bac}+3$ (Licences professionnelles portées par les IUT de Cachan et Ville d'Avray, notamment) au Bac +5 (Ecole d'ingénieur spécialisée dans l'embarquée). Ce rééquilibrage s'est fait au détriment des formations en réseaux qui avaient auparavant le vent en poupe.

Ainsi, une réflexion complémentaire est nécessaire. Le projet est sans doute trop long. Commencé tôt, afin de motiver les étudiants, il a fini tard pour profiter des acquis. Il faut certainement resserrer ce calendrier. D'autre part, l'obtention de résultats intermédiaires fonctionnels (carte de vision, hacheur, simulation fonctionnelle...) devrait être plus valorisée.

S'il y avait une recette miracle pour lutter contre l'échec en premier cycle, elle serait connue depuis longtemps. Ce constat n'implique pas, bien au contraire, qu'il faille baisser les bras, les étudiants étant très sensibles aux efforts que produisent leurs formateurs.

\section{Bibliographie}

[1] Gilles Raynaud, "Pédagogies pour la réussite en IUT", Actes du Colloques du GESI (Groupement des enseignant en IUT GEII), Lyon (France), Décembre 2005, pp. 4-8.

[2] Bill Gates, "Un robot dans chaque foyer", Pour la science, $n^{\circ} 356$ (Juin 2007), pp. 76-81.

[3] Collectif, "Reglement du concours de robotique des IUT GEII", disponible en téléchargement à l'adresse : http://web.geii.univsavoie.fr/geii/robots/reglement Vierzon 2008 R007.pdf

[4] Dominique Nardi, "robot opto guidé", article du site iut en ligne disponible à l'adresse : http://www.iutenligne.net/ressources/etudes re alisations/Nardi/Robot/ 